\title{
Hemipelvic osteomyelitis in a hemodialysis patient associated with methicillin-resistant Staphylococcus aureus bacteremia
}

\author{
Jeanne C Patzkowski \\ Frank P Hurst ${ }^{1,2}$ \\ Robert T Neff ${ }^{1,2}$ \\ Kevin C Abbott ${ }^{1,2}$ \\ 'Uniformed Services University \\ of the Health Sciences F. Edward \\ Hebert School of Medicine, Bethesda, \\ MD, USA; ${ }^{2}$ Nephrology Service, \\ Walter Reed Army Medical Center, \\ Washington, DC, USA
}

\begin{abstract}
Proper management of infected tunneled-cuffed catheters (TCC) is essential in order to avoid catastrophic consequences for the patient. Hematogenous dissemination of infection can result in serious secondary infections, including infective endocarditis, osteomyelitis, and epidural abscess. Pelvic osteomyelitis is an extremely rare condition in adults with no reported cases of infection localized to more than one pelvic bone at a time. We present a case of a hemodialysis patient who developed osteomyelitis of the entire right hemipelvis due to MRSA bacteremia after repeated attempts at TCC salvage.
\end{abstract}

Keywords: pelvic osteomyelitis, indwelling catheter, staphylococcus aureus, catheter-related bacteremia

\section{Introduction}

The management of infected tunneled-cuffed-catheters (TCC) is complicated, and the question of whether to salvage, replace, or remove infected catheters has been extensively debated. ${ }^{1-7}$ Regardless, appropriate management of catheter-related bacteremia (CRB) is essential, because the consequences can be severe. Aggressive treatment may be required to avoid hematogenous complications such as infective endocarditis, osteomyelitis, and epidural abscesses. ${ }^{1}$

Hematogenous osteomyelitis in the adult usually localizes to the bones of the axial skeleton, most commonly the vertebral bodies and intervertebral discs. ${ }^{2}$ A literature review revealed few cases of pelvic osteomyelitis in adults. While infections of the pubic symphysis, sacro-iliac joints, and ischia have been reported in adults, they are usually associated with specific risk factors such as uro-gynecological surgery, mycobacterial infection, and trauma. ${ }^{2,8-13}$

We report a case of osteomyelitis of the entire right hemipelvis in a hemodialysis (HD) dependent patient with methicillin-resistant Staphylococcus aureus (MRSA) bacteremia secondary to TCC infection after repeated attempts at catheter salvage.

\section{Case report}

A 26-year-old Caucasian male with end stage renal disease (ESRD) on hemodialysis was referred to our outpatient clinic to be evaluated for renal transplantation. He had been initiated on HD 6 months previously, after presenting with ESRD. Renal biopsy at that time showed extensive glomerulosclerosis. His medical history was remarkable for a childhood seizure disorder, chronic lower back pain attributed to an L5-S1 herniated disk, and an allergy to penicillin. He denied a history of intravenous drug abuse and his only surgery had been the placement of a TCC for HD. His medications included carvedilol, sevelamer, nifedipine, and oxycodone/acetaminophen. 
On initial examination his blood pressure was $128 / 68 \mathrm{mmHg}$ and heart rate was 111 beats per minute. He was febrile to $102.5^{\circ} \mathrm{F}\left(39.2^{\circ} \mathrm{C}\right)$. His physical exam was remarkable for crackles at the left lung base, a grade III/VI systolic ejection murmur, anasarca, and localized erythema about the TCC insertion without frank purulence or discharge. Laboratories demonstrated a white blood cell count $17.8 \times 10^{3} / \mu \mathrm{L}\left(18 \times 10^{9} / \mathrm{L}\right)$, hemoglobin $7.1 \mathrm{~g} / \mathrm{dL}$ (71 g/L), blood urea nitrogen $32 \mathrm{mg} / \mathrm{dL}$ (11.4 mmol/L), creatinine $5.19 \mathrm{mg} / \mathrm{dL}(396 \mu \mathrm{mol} / \mathrm{L})$, alkaline phosphatase (AP) $158 \mathrm{U} / \mathrm{L}$, erythrocyte sedimentation rate $86 \mathrm{~mm} / \mathrm{hr}$ (normal < 19), and C-reactive protein (CRP) $4.99 \mathrm{mg} / \mathrm{dL}$ (normal $<0.50$ ). Blood cultures grew $S$. aureus resistant to oxacillin (MRSA) in all bottles and vancomycin therapy was initiated followed by removal of the TCC.

Additional history revealed multiple "changes" of his HD catheter over the preceding 4 months for "line infections". It is unclear if these attempts at catheter salvage involved wire-changes, but the patient denied a catheter-free interval or change of catheter location during that period.

Two months previously, he had reported progressive back and right hip pain with ambulation. Radiographs of the right hip and femur demonstrated no bony or soft tissue abnormalities, and an MRI of the lumbar spine demonstrated a disc herniation at L5-S1 with apparent compression of the right S1 nerve root. Gradually he developed weakness of the right lower extremity and one month later was wheelchair dependent. He denied paresthesias or incontinence, and because of the progressive nature of his symptoms, was scheduled for an elective laminectomy before renal transplant.

On arrival at our institution, the neurosurgery department was consulted to assess his pain and weakness and to evaluate for epidural abscess given the known disk herniation in the setting of bacteremia. They concluded that the disc herniation was an incidental finding unrelated to his presentation. Concomitant radiographs (Figure 1A), computed tomography (Figure 1B), and magnetic resonance imaging (MRI) demonstrated gross osteomyelitis and pyoarthrosis involving the right ilium, acetabulum, pubis, and ischium with severe edema and multiple abscesses throughout the soft tissue with no involvement of the left hemipelvis or sacroiliac joints.

Several other foci of hematogenous infection were identified during his admission including septic pulmonary emboli, ventriculitis, and neuroretinitis, although trans-esophageal

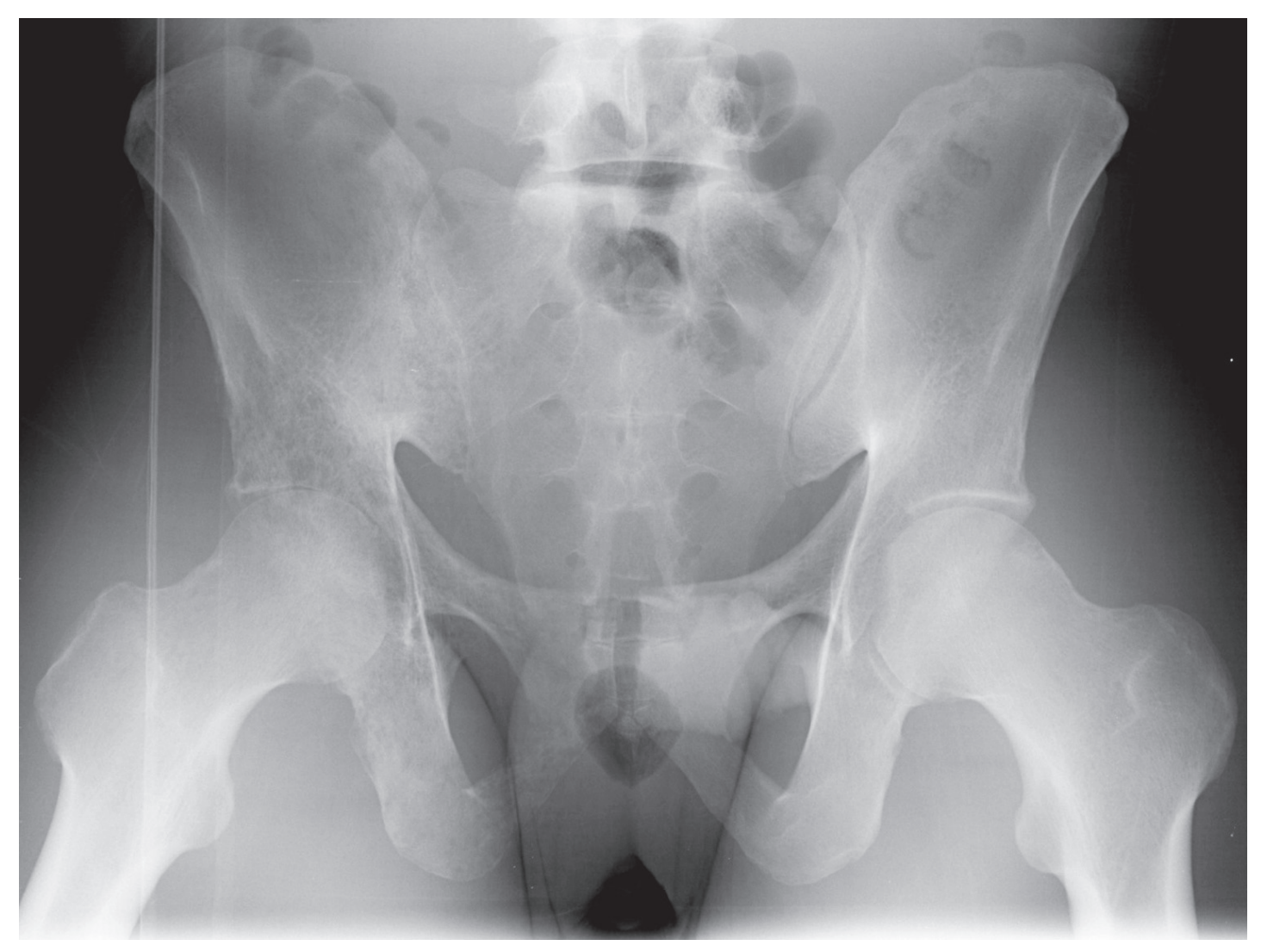

Figure I Pelvic radiograph. This pre-treatment radiograph demonstrates a diffuse lytic process within the right iliac wing extending into the acetabulum and right pubic rami. 
echocardiography demonstrated no evidence of infective endocarditis. Hip joint aspirations were nondiagnostic with negative gram stain and culture, but given the blood cultures and multiple foci of hematogenous dissemination, MRSA was presumed to be the causative organism. Given the extent of the infection, surgical debridement was not favored as an option as it would necessitate a right hemipelvectomy.

The TCC was replaced once his blood cultures had cleared, and on the advice of the Infectious Disease service, rifampin was added to the vancomycin. After 4 weeks of therapy his functional status vastly improved, and he was able to ambulate with the use of orthotics. He was eventually discharged from the hospital with vancomycin to be dosed at HD. Repeat CT scan after 6 weeks of therapy demonstrated spread of osteomyelitis to the right sacroiliac joint, but overall improvement and resolution of the abscesses. At that time, his labs demonstrated a decrease in AP to $91 \mathrm{U} / \mathrm{L}$ and down trending CRP of $3.78 \mathrm{mg} / \mathrm{dL}$.

\section{Discussion}

Bacteremia is a common complication resulting from the use of TCC with an incidence of approximately 3 per 1000 catheter-days. ${ }^{1,3-5}$ Secondary hematogenous infections such as endocarditis, osteomyelitis, and epidural abscess have especially high costs in morbidity and mortality, and typically require aggressive treatment measures. ${ }^{1}$ Nissenson et al found that osteomyelitis occurred in $1.9 \%$ of HD patients admitted with S. aureus septicemia and was associated with a significantly increased length of stay (23.9 vs 13.3 days; $p<0.05)$ and Medicare cost ( $\$ 26,725$ vs $\$ 18,476 ; p<0.05)$, compared with bacteremic patients with no complications. ${ }^{14}$ Four independent risk factors (MRSA bacteremia, permanent foreign body, HD dependence, and a delay or failure to remove a central venous catheter) have been associated with hematogenous complications of CRB. ${ }^{2}$ While society guidelines for CRB offer catheter salvage as an option in certain patients, ${ }^{6,7}$ salvage therapy frequently fails to clear the infection, especially with MRSA. ${ }^{3}$ Additionally, S. aureus infection has been found to be an independent risk factor for TCC salvage failure and the development of metastatic infections. ${ }^{3,8,15}$

Recent studies suggest that TCC salvage has a high failure rate in bacteremic patients, because systemic

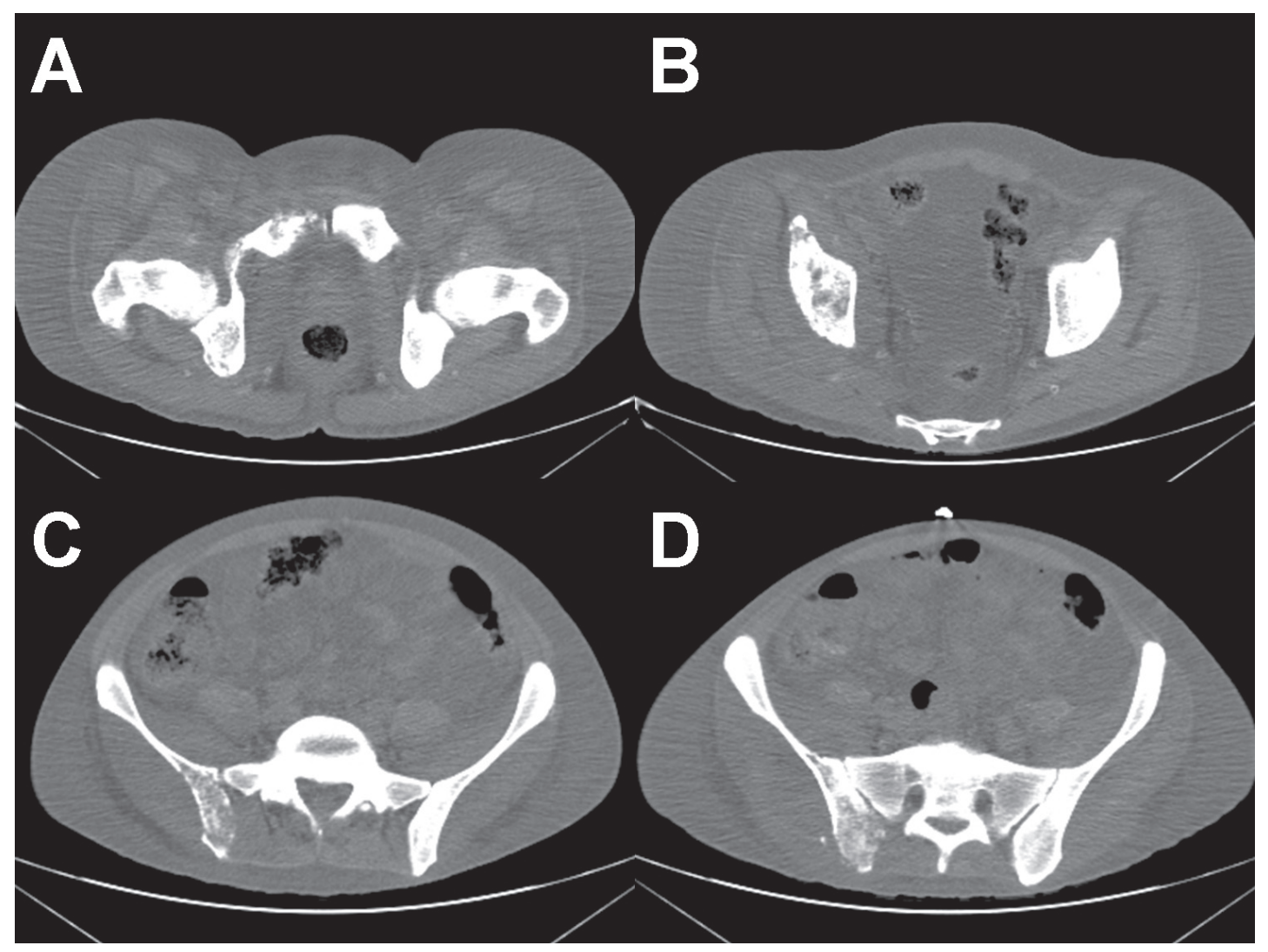

Figure 2 (A-D) Computed tomography of pelvis. These pre-treatment CT images demonstrate diffuse bony destruction with loss of normal cortical contour and trabeculations within the posterior aspect of the right ilium, right acetabulum, and right symphysis pubis. 
antibiotics are unable to penetrate the biofilm coating the catheter lumen. ${ }^{3}$ Substituting the catheter for a guidewire appears to be a reasonable alternative given that the site is preserved, the biofilm removed, and a decreased incidence of treatment failure and serious secondary infections have been described. ${ }^{1}$

Our patient reported "multiple changes" of his TCC, and it is possible that his initial infection never cleared. Further attempts at salvage should have been abandoned in favor of catheter removal with replacement in a different site. Unfortunately, as demonstrated here, the consequences of $\mathrm{CRB}$ and failed salvage can be catastrophic.

Osteomyelitis of the pelvis is not uncommon in the pediatric population, but is extremely rare in adults, with most infections affecting the pubic symphysis or sacro-iliac joints. These cases comprise less than $1 \%$ of all cases of hematogenous osteomyelitis. ${ }^{8}$ Most cases of pelvic osteomyelitis reported demonstrate infection isolated to single pelvic bones with distinct risk factors for each site. For example, infection of the pubic symphysis is generally a complication of uro-gynecological surgery, prolonged bladder catheterization, pregnancy, IVDA, trauma, diabetes, or intense athletic competition. ${ }^{2,8-12}$ Infection of the ischium is most commonly due to Mycobacteria with one case of pseudomonal infection reported after radical bladder surgery, and infection of the ilium has been reported in bacteremic children, with no reports in adults. ${ }^{2,13,16-18}$ Osteomyelitis simultaneously infecting multiple bones of the pelvis is rare, but has been reported in children. ${ }^{19}$

Based on the time course of the patient's symptoms, the diagnosis of osteomyelitis could have been delayed, and the extent of disease may represent contiguous spread of the original focus of infection. Pelvic osteomyelitis is often characterized by vague symptoms of poorly defined hip pain, limited range of motion, and difficulty with ambulation. ${ }^{15,19}$ Furthermore, the MRI of the lumbar spine could have confounded the clinical picture by providing an alternative explanation for his symptoms.

Due to its low incidence, pelvic osteomyelitis is not routinely considered in the differential diagnosis of an adult with hip pain. However, as demonstrated by this patient, the consequences of a delayed diagnosis can be severe. We recommend that pelvic osteomyelitis be considered in any hemodialysis patient with progressive, intractable hip or back pain in the setting of CRB. This case also emphasizes that failed catheter salvage techniques can have catastrophic consequences and should be used with caution and possibly not at all in all patients with MRSA CRB.

\section{Acknowledgments}

The authors wish to thank all hospital staff and consulting services who were involved in the care of this patient. The views expressed in this paper are those of the authors and do not reflect the official policy of the Department of Army, Department of Defense, or the US government. The authors are employees of the United States government. This work was prepared as part of their official duties and as such, there is no copyright to be transferred. The authors report no conflicts of interest.

\section{References}

1. Philipneria M, Al-Aly, ZA, Amin K, et al. Routine replacement of tunneled, cuffed, hemodialysis catheters eliminates paraspinal/ vertebral infections in patients with catheter-associated bacteremia. Am J Nephrol. 2003;23:202-7.

2. Fowler VG, Justice A, Moore C, et al. Risk factors for hematogenous complications of intravascular catheter-associated Staphylococcus aureus bacteremia. Clin Infect Dis. 2005;40:695-703.

3. Mokrzycki MH, Zhang M, Cohen H, et al. Tunnelled haemodialysis catheter bacteraemia: risk factors for bacteraemia recurrence, infectious complications and morality. Nephro Dial Transplant. 2006;21:1024-31.

4. Marr KA, Sexton DJ, Conlon PJ, et al. Catheter-related bacteremia and outcome of attempted catheter salvage in patients undergoing hemodialysis. Ann Intern Med. 1997;127:275-80.

5. Kovalik EC, Raymond JR, Albers FJ, et al. A clustering of epidural abscesses in chronic hemodialysis patients; risks of salvaging access catheters in cases of infection. J Am Soc Nephrol. 1996;7:2264-7.

6. NKF K/DOQI ${ }^{\mathrm{TM}}$ Guidelines: Clinical Practice Guidelines and Clinical Practice Recommendations 2006 Updates, National Kidney Foundation, Inc., 2006.

7. Mermel LA, Farr BM, Sherertz RJ, et al. Guidelines for the Management of Intravascular Catheter-Related Infections. Clin Infect Dis. 2001;32:1249.

8. Dourakis SP, Alexopoulou A, Metallinos G, et al. Pubic osteomyelitis due to klebsiella pneumoniae in a patient with diabetes mellitus. Am J Med Sci. 2006;331:322-4.

9. Karpos PAG, Spindler KP, Pierce MA, et al. Osteomyelitis of the pubic symphysis in athletes: a case report and literature review. Med Sci Sports Exerc. 1995;27:473-8.

10. Enzler M, Agins HJ, Kogan M, et al. Osteomyelitis of the pubic following suspension of the neck of the bladder with use of bone anchors. A report of four cases. J Bone Joint Surg Am. 1999;81:1736-40.

11. Stern JA, Clemens JQ. Osteomyelitis of the pubis; a complication of a chronic indwelling catheter. Urology. 2003;61:462.

12. Ross JJ, Hu LT. Septic arthritis of the pubic symphysis: Review of 100 cases. Medicine. 2003;82:340-5.

13. Berthelot JM, Varin S, Caillon F, et al. Pseudomonas aeruginosa osteomyelitis of both ischia. J Bone Joint Surg Am. 2002;84:441-4.

14. Nissenson AR, Dylan ML, Griffiths RI, et al. Clinical and economic outcomes of Staphylococcus aureus septicemia in ESRD patients receiving hemodialysis. Am J Kidney Dis. 2005;46:301-8.

15. Sia IG, Berbari EF. Osteomyelitis. Best Prac Res Clin Rheum. 2006;20:1065-81.

16. Beaupre A, Carroll N. The three syndromes of iliac osteomyelitis in children. J Bone Joint Surg Am. 1979;61-A:1087-92.

17. Chung SMK, Borns P. Acute osteomyelitis adjacent to the sacroiliac joint in children: Report of two cases. J Bone Joint Surg Am. 1973;55:630-4.

18. Abbot AE, Sculco TP. Septic sacroiliitis with hematogenous spread to a total knee arthroplasty. J Arthroplasty. 2001;16:225-8.

19. Weber-Chrysochoou C, Corti N, Goetschel P, et al. Pelvic osteomyelitis: a diagnostic challenge in children. J Ped Surg. 2007;42:553-7. 